\title{
Robust Adaptive Attitude Control for Airbreathing Hypersonic Vehicle with Attitude Constraints and Propulsive Disturbance
}

\author{
Jian Fu, ${ }^{1}$ Liangming Wang, ${ }^{1}$ Mou Chen, ${ }^{2}$ and Sijiang Chang ${ }^{1}$ \\ ${ }^{1}$ College of Energy and Power Engineering, Nanjing University of Science and Technology, Nanjing 210094, China \\ ${ }^{2}$ College of Automation Engineering, Nanjing University of Aeronautics and Astronautics, Nanjing 210016, China \\ Correspondence should be addressed to Jian Fu; fujian@njust.edu.cn
}

Received 16 July 2015; Accepted 26 November 2015

Academic Editor: Marek Lefik

Copyright (C) 2015 Jian Fu et al. This is an open access article distributed under the Creative Commons Attribution License, which permits unrestricted use, distribution, and reproduction in any medium, provided the original work is properly cited.

A robust adaptive backstepping attitude control scheme, combined with invariant-set-based sliding mode control and fastnonlinear disturbance observer, is proposed for the airbreathing hypersonic vehicle with attitude constraints and propulsive disturbance. Based on the positive invariant set and backstepping method, an innovative sliding surface is firstly developed for the attitude constraints. And the propulsive disturbance of airbreathing hypersonic vehicle is described as a differential equation which is motivated by attitude angles in this paper. Then, an adaptive fast-nonlinear disturbance observer for the proposed sliding surface is designed to estimate this kind of disturbance. The convergence of all closed-loop signals is rigorously proved via Lyapunov analysis method under the developed robust attitude control scheme. Finally, simulation results are given to illustrate the effectiveness of the proposed attitude control scheme.

\section{Introduction}

In recent years, a new kind of aerospace vehicle, which is called airbreathing hypersonic vehicle (AHV), has attracted the considerable attention in both military and civil communities. Comparing with conventional flight vehicles, this new aerospace vehicle can sustain flight for a long time with high flight speed and cover a large envelope $[1,2]$. Due to the strong disturbance and fast changed pneumatics parameters, it is still a challenging task to design a robust AHV control system. Therefore, considerable efforts have been made to develop an efficient flight controller for this kind of aerospace vehicles. In [3, 4], adaptive function link network control and fuzzy logical system were proposed for the aerospace vehicle with uncertainties. And baseline nonlinear model predictive controller was presented in [5] for AHV with nonvanishing mismatched disturbances. In [6], an adaptive fault-tolerant control strategy with input saturation is proposed for AHV flight system with actuator faults and external disturbance. However, the robust attitude control method should be further developed for the AHV with attitude constraints and propulsive disturbance.

In order to suppress the unknown external disturbance from changeable flight environment, many papers have focused on the disturbance observer of AHV flight control system [7-11]. A nonlinear disturbance observer based control (NOBC) method is proposed in [7] for the longitudinal dynamics of a generic airbreathing hypersonic vehicle under mismatched disturbance. In [8], the NDO technique is combined with Takagi-Sugeno (T-S) fuzzy linear model to design a disturbance observer based guaranteed cost fuzzy controller (GCFC) for the AHV flight systems. To achieve satisfactory tracking performance, a composite tracking controller is proposed for AHV using NDO and backstepping technologies [9]. However, there are few disturbance observer based control results for AHV with the propulsive disturbance. This kind of disturbance for AHV is usually motivated from the coupling between the scramjet propulsion system and attitude angles [10, 11]. Thus, this kind of disturbance will lead to a challenging problem for 
the attitude control of the AHV. In this paper, the propulsive disturbance is described as a differential equation which is motivated by attitude constraints in this paper. And a composite controller is proposed to handle this disturbance using adaptive NDO and backstepping technologies.

Furthermore, sliding mode control (SMC) is considered as an important method due to its high precision control, relative simplicity of control design, and high robust features with respect to system internal perturbations and external disturbances. Thus, the SMC and its application of AHV have been widely studied in [12-21]. A composite control treatment based on sliding model method is presented to the nonlinear longitudinal dynamics of AHV vehicles [12]. And dynamic terminal sliding mode technique is proposed for the robust control design of $\operatorname{AHV}[13,14]$. Considering the input constraints, a second-order terminal sliding mode control method is discussed for AHV flight system in reentry phase [15]. Meanwhile, an adaptive dynamic sliding mode control method is presented for the fault-tolerant control problem of AHV in [16]. However, most of the AHV control systems combining backstepping control with SMC have not considered the attitude constraints. Thus, it is necessary to design an innovative sliding mode control for the AHV using backstepping technique and considering the attitude constraints.

This work is motivated by the robust attitude control of AHV with attitude constraints and propulsive disturbance. The control objective is that the proposed robust control scheme can track a desired trajectory in the presence of the unknown propulsive disturbance and attitude constraints. An innovative sliding surface is firstly developed in this paper. And the invariant sets and saturation function are utilized in this surface for the attitude constraints of AHV. To guarantee the control effects under propulsive disturbance, the switching information from invariant-set-based sliding surface is included in the adaptive disturbance observer to increase the convergence speed. Therefore, the organization of the paper is as follows. Section 2 details the problem formulation, while main results are given in Section 3. Simulation results are presented in Section 4 to show the effectiveness of proposed robust adaptive backstepping attitude control for AHV. And some conclusion results are shown in Section 5.

\section{Problem Statement}

2.1. Model Description. To study the robust attitude controller, a nonlinear attitude motion model of AHV flight system is described as the following nonlinear affine nonlinear system [22-24]:

$$
\begin{aligned}
\dot{\mathbf{x}}_{1} & =\mathbf{F}_{s}\left(\mathbf{x}_{1}\right)+\mathbf{G}_{s}\left(\mathbf{x}_{1}\right) \mathbf{x}_{2}, \\
\dot{\mathbf{x}}_{2} & =\mathbf{F}_{f}\left(\mathbf{x}_{1}, \mathbf{x}_{2}\right)+\mathbf{G}_{f}\left(\mathbf{x}_{1}, \mathbf{x}_{2}\right) \mathbf{u}+\mathbf{d}(t), \\
\mathbf{y} & =\mathbf{x}_{1},
\end{aligned}
$$

where $\mathbf{x}_{1}=[\alpha, \beta, \mu]^{T} \in R^{3}$ is the attitude angle vector of slowloop states, $\mathbf{x}_{2}=[p, q, r]^{T} \in R^{3}$ is the body-axis angular rate vector of fast-loop states, $\mathbf{u}=\left[\delta_{e}, \delta_{a}, \delta_{r}, \delta_{x}, \delta_{y}, \delta_{z}\right]^{T} \in R^{6}$ is the deflection vector of control surfaces which are the system control inputs, $y \in R^{3}$ is the system output, and the unknown disturbance $\mathbf{d}\left(\mathbf{y}, \mathbf{y}_{d}, t\right) \in R^{3}$ is defined as

$$
\mathbf{d}\left(\mathbf{y}, \mathbf{y}_{d}, t\right)=\mathbf{d}_{1}\left(\mathbf{y}, \mathbf{y}_{d}, t\right)+\mathbf{d}_{2}(t),
$$

where $\mathbf{d}_{1}\left(\mathbf{y}, \mathbf{y}_{d}, t\right) \in R^{3}$ is the unknown propulsive disturbance which is coupling with the attitude angles and $\mathbf{d}_{2}(t) \in R^{3}$ is the rest of unknown disturbance. The detailed expression of $\mathbf{F}_{s} \in R^{3}, \mathbf{G}_{s} \in R^{3 \times 3}, \mathbf{F}_{f} \in R^{3}$, and $\mathbf{G}_{f} \in R^{3 \times 6}$ can be found in [24].

2.2. Assumptions and Control Objective. In order to reduce the coupling effects between the scramjet propulsion system and attitude angles, the attitude constraints are defined as

$$
\begin{aligned}
\Psi & =\left\{\mathbf{y} \in R^{3} \mid y_{d}^{(i)}-c_{i} \leq y_{i} \leq y_{d}^{(i)}+c_{i}, c_{i} \in R^{+}, i\right. \\
& =1,2,3\},
\end{aligned}
$$

where $\mathbf{y}=\left[y_{1}, y_{2}, y_{3}\right]^{T}$ is the attitude angle vector and $\mathbf{y}_{d}=\left[y_{d}^{(1)}, y_{d}^{(2)}, y_{d}^{(3)}\right]^{T}$ is the desired output signal. When the attitude angle vector $\mathbf{y}$ satisfies constraints (3), the propulsive disturbance $\mathbf{d}_{1}(\mathbf{y}, t)$ will be a small value which can be neglected in the control design process. However, if y exceeds the boundary of constraints (3), this disturbance will be increased significantly. Then, it is supposed that the disturbances $\mathbf{d}_{1}(\mathbf{y}, t)$ and $\mathbf{d}_{2}(t)$ will satisfy the following assumptions.

Assumption 1. For all $t>0$, there exists $\Delta_{i}$ such that $\left\|y_{d}^{(i)}(t)\right\| \leq \Delta_{i}, i=1,2,3$.

Assumption 2. The propulsive disturbance $\mathbf{d}_{1}\left(\mathbf{y}, \mathbf{y}_{d}, t\right)$ can be described as the following differential equation:

$$
\dot{\mathbf{d}}_{1}\left(\mathbf{y}, \mathbf{y}_{d}, t\right)=\tau_{1}(\mathbf{y}, t) \cdot \boldsymbol{\delta}_{1 \varepsilon}\left(\mathbf{y}, \mathbf{y}_{d}\right),
$$

where $\boldsymbol{\tau}_{1}(\mathbf{y}, t)=\operatorname{diag}\left\{\tau_{11}, \tau_{12}, \tau_{13}\right\}$, and we can obtain $\boldsymbol{\tau}_{1}(\mathbf{y}, t)=[0,0,0]^{T}$ if $\mathbf{y} \in \psi$. For the element $\tau_{1 i}$, there exists an unknown positive constant $\kappa_{i}$ such that $\left\|\tau_{1 i}\right\| \leq \kappa_{i}, i=1,2,3$. The vector $\boldsymbol{\delta}_{1 \varepsilon} \in R^{3}$ is a special designed sliding surface as shown in (22), and there exists $\mathbf{y} \in \boldsymbol{\Psi}$ while $\boldsymbol{\delta}_{1 \varepsilon}=0$.

Assumption 3. The disturbance $\mathbf{d}_{2}(t)$ can be described as the following differential equation:

$$
\dot{\mathbf{d}}_{2}(t)=\boldsymbol{\tau}_{2}(t),
$$

where $\boldsymbol{\tau}_{2}(t)=\left[\tau_{21}, \tau_{22}, \tau_{23}\right]^{T}$, and there exists a known positive constant $\mu_{i}$ such that $\left\|\tau_{2 i}(t)\right\| \leq \mu_{i}, i=1,2,3$.

Assumption 4. The generalized matrix inverses of $\mathbf{G}_{s}$ and $\mathbf{G}_{f}$ are always existing for the nonlinear attitude motion model of the AHV, and the elements in matrixes $\mathbf{F}_{s}, \mathbf{G}_{s}, \mathbf{F}_{f}$, and $\mathbf{G}_{f}$ are always continuous for the nonlinear attitude motion model of the AHV.

Definition 1 (see [25]). The set $\mathbf{Q}$ is said to be positively invariant (PI) for nonlinear system (1), if, for all $\mathbf{x}_{1}\left(t_{1}\right) \in \mathbf{Q}$, there exists the solution $\mathbf{x}_{1}\left(t_{2}\right) \in \mathbf{Q}, \forall t_{2}>t_{1}$. 


\section{Robust Adaptive Backstepping Attitude Control for the AHV with Attitude Constraints and Propulsive Disturbance}

3.1. Preliminary. In this section, a few results are given to support the design process of robust adaptive backstepping attitude controller. Considering the standard backstepping control design method, we define

$$
\begin{aligned}
& \mathbf{e}_{1}=\mathbf{y}-\mathbf{y}_{d}, \\
& \mathbf{e}_{2}=\mathbf{x}_{2}-\boldsymbol{v}_{1},
\end{aligned}
$$

where $\nu_{1} \in R^{3}$ is a designed virtual control law. Then, considering (1), the derivatives $\dot{\mathbf{e}}_{1}, \dot{\mathbf{e}}_{2}$ can be written as

$$
\begin{aligned}
& \dot{\mathbf{e}}_{1}=\mathbf{F}_{s}\left(\mathbf{x}_{1}\right)+\mathbf{G}_{s}\left(\mathbf{x}_{1}\right) \cdot\left(\mathbf{e}_{2}+\boldsymbol{v}_{1}\right)-\dot{\mathbf{y}}_{d}, \\
& \dot{\mathbf{e}}_{2}=\mathbf{F}_{f}\left(\mathbf{x}_{1}, \mathbf{x}_{2}\right)+\mathbf{G}_{f}\left(\mathbf{x}_{1}, \mathbf{x}_{2}\right) \cdot \mathbf{u}+\mathbf{d}\left(\mathbf{y}, \mathbf{y}_{d}, t\right)-\dot{\boldsymbol{v}}_{1},
\end{aligned}
$$

where $\mathbf{e}_{1}=\left[e_{11}, e_{12}, e_{13}\right]^{T} \in R^{3}$ are the state errors of slowloop states and $\mathbf{e}_{2}=\left[e_{21}, e_{22}, e_{23}\right]^{T} \in R^{3}$ are the state errors of fast-loop states. Invoking (6), the attitude constraints (3) can be rewritten as

$$
\Psi=\left\{\left(\mathbf{e}_{1}, \int \mathbf{e}_{1}\right) \mid-c_{i} \leq e_{1 i} \leq c_{i}, c_{i} \in R^{+}\right\}
$$

where $\Psi=\left[\Psi_{1}, \Psi_{2}, \Psi_{3}\right]^{T}$ and $\Psi_{i}=\left\{\left(e_{1 i}, \int e_{1 i}\right) \mid-c_{i} \leq e_{1 i} \leq c_{i}\right\}$.

Considering the attitude constraints of AHV, the unidirectional auxiliary surfaces (UAS) for slow-loop and fastloop states are utilized to design the invariant-set-based sliding mode controller. The detailed design process for these surfaces is given as follows.

Step 1. Considering the slow-loop system (7), we define the switching surfaces as

$$
\begin{aligned}
& \mathbf{S}_{11}=\mathbf{e}_{1}+\xi_{11} \cdot \int \mathbf{e}_{1}=0, \\
& \mathbf{S}_{12}=\mathbf{e}_{1}+\xi_{12} \cdot \int \mathbf{e}_{1}=0,
\end{aligned}
$$

where $\xi_{11}=\operatorname{diag}\left\{\xi_{111}, \xi_{112}, \xi_{113}\right\}, \xi_{12}=\operatorname{diag}\left\{\xi_{121}, \xi_{122}\right.$, $\left.\xi_{123}\right\}, \mathbf{S}_{11}=\left[S_{111}, S_{112}, S_{113}\right]^{T}, \mathbf{S}_{12}=\left[S_{121}, S_{122}, S_{123}\right]^{T}, \xi_{11 i}>$ $\xi_{12 i}>0, i=1,2,3$, and $\int \mathbf{e}_{1} d t$ is denoted by $\int \mathbf{e}_{1}$ for brevity. The conditions $\xi_{11 i}>0$ and $\xi_{12 i}>0$ are given to guarantee the stability of switching surfaces $S_{11 i}=0$ and $S_{12 i}=0$. And the condition $\xi_{11 i}>\xi_{12 i}$ is used to avoid the overlap of switching surfaces.

Step 2. Invoking the coefficients $\xi_{11 i}, \xi_{12 i}$ in (10), the unidirectional auxiliary surface $h_{1 i}$ can be designed as

$$
h_{1 i}=\omega_{1 i 1} \cdot e_{1 i}+\omega_{1 i 2} \cdot \int e_{1 i}+M_{1 i}
$$

where

$$
\begin{gathered}
\omega_{1 i 1}= \begin{cases}1 & S_{11 i}<0 ; S_{12 i}<0 \\
\omega_{11 i 1} & S_{11 i}<0 ; S_{12 i} \geq 0 \\
\omega_{12 i 1} & S_{11 i} \geq 0 ; S_{12 i}<0 \\
-1 & S_{11 i} \geq 0 ; S_{12 i} \geq 0 ;\end{cases} \\
\omega_{1 i 2}= \begin{cases}\omega_{10 i 2} & S_{11 i}<0 ; S_{12 i}<0 \\
\omega_{11 i 2} & S_{11 i}<0 ; S_{12 i} \geq 0 \\
\omega_{12 i 2} & S_{11 i} \geq 0 ; S_{12 i}<0 \\
-\omega_{13 i 2} & S_{11 i} \geq 0 ;\end{cases}
\end{gathered}
$$

where $\omega_{10 i 2}, \omega_{13 i 2}$ are the designed parameters which satisfy $\xi_{12 i}<\omega_{10 i 2}<\xi_{11 i}, \xi_{12 i}<\omega_{13 i 2}<\xi_{11 i}$, and $M_{1 i}>0$, and the rest of coefficients are given as follows:

$$
\begin{aligned}
& \omega_{11 i 1}=\frac{\left[-\left(\xi_{11 i}+\xi_{12 i}\right)+\left(\omega_{10 i 2}+\omega_{13 i 2}\right)\right]}{\left(\xi_{11 i}-\xi_{12 i}\right)}, \\
& \omega_{11 i 2}=\frac{\left[-2 \xi_{11 i} \xi_{12 i}+\left(\omega_{10 i 2} \xi_{11 i}+\omega_{13 i 2} \xi_{12 i}\right)\right]}{\left(\xi_{11 i}-\xi_{12 i}\right)}, \\
& \omega_{12 i 1}=\frac{\left[\left(\xi_{11 i}+\xi_{12 i}\right)-\left(\omega_{10 i 2}+\omega_{13 i 2}\right)\right]}{\left(\xi_{11 i}-\xi_{12 i}\right)}, \\
& \omega_{12 i 2}=\frac{\left[2 \xi_{11 i} \xi_{12 i}-\left(\omega_{13 i 2} \xi_{11 i}+\omega_{10 i 2} \xi_{12 i}\right)\right]}{\left(\xi_{11 i}-\xi_{12 i}\right)},
\end{aligned}
$$

where $\omega_{11 i 1} \neq 0$ and $\omega_{12 i 1} \neq 0$. Then, the compact form of unidirectional auxiliary surface

$$
\mathbf{h}_{1}=\mathbf{\Omega}_{11} \cdot \mathbf{e}_{1}+\mathbf{\Omega}_{12} \cdot \int \mathbf{e}_{1}+\mathbf{M}_{1}
$$

where $\mathbf{h}_{1}=\left[h_{11}, h_{12}, h_{13}\right]^{T}, \int \mathbf{e}_{1}=\left[\int e_{11}, \int e_{12}, \int e_{13}\right]^{T}$, $\boldsymbol{\Omega}_{11}=\operatorname{diag}\left\{\omega_{111}, \omega_{121}, \omega_{131}\right\}, \boldsymbol{\Omega}_{12}=\operatorname{diag}\left\{\omega_{112}, \omega_{122}, \omega_{132}\right\}$, and $\mathbf{M}_{1}=\left[M_{11}, M_{12}, M_{13}\right]^{T}$ is a constant vector.

As shown in Figure 1, the UAS can form a convex set $Q_{i}$ which satisfies $Q_{i} \subseteq \Psi_{i}$, and the expression of set $Q_{i}$ is written as

$$
Q_{i}=\left\{\left(e_{1 i}, \int e_{1 i}\right) \mid h_{1 i} \geq 0\right\} .
$$

The compact form of convex set $Q_{i}$ can be written as $\mathbf{Q}=$ $\left[Q_{1}, Q_{2}, Q_{3}\right]^{T}$, where $\mathbf{Q} \subseteq \Psi$ implies $Q_{i} \subseteq \Psi_{i}, \quad i=1,2,3$.

Step 3. Similarly, we can define the following switching surfaces for the fast-loop system (8):

$$
\begin{aligned}
& \mathbf{S}_{21}=\mathbf{e}_{2}+\boldsymbol{\xi}_{21} \cdot \int \mathbf{e}_{2}=0, \\
& \mathbf{S}_{22}=\mathbf{e}_{2}+\boldsymbol{\xi}_{22} \cdot \int \mathbf{e}_{2}=0,
\end{aligned}
$$

where $\boldsymbol{\xi}_{21}=\operatorname{diag}\left\{\xi_{211}, \xi_{212}, \xi_{213}\right\}, \boldsymbol{\xi}_{22}=\operatorname{diag}\left\{\xi_{221}, \xi_{222}, \xi_{223}\right\}$, $\mathbf{S}_{21}=\left[S_{211}, S_{212}, S_{213}\right]^{T}, \mathbf{S}_{22}=\left[S_{221}, S_{222}, S_{223}\right]^{T}$, and $\xi_{21 i}>$ $\xi_{22 i}>0, i=1,2,3$. 


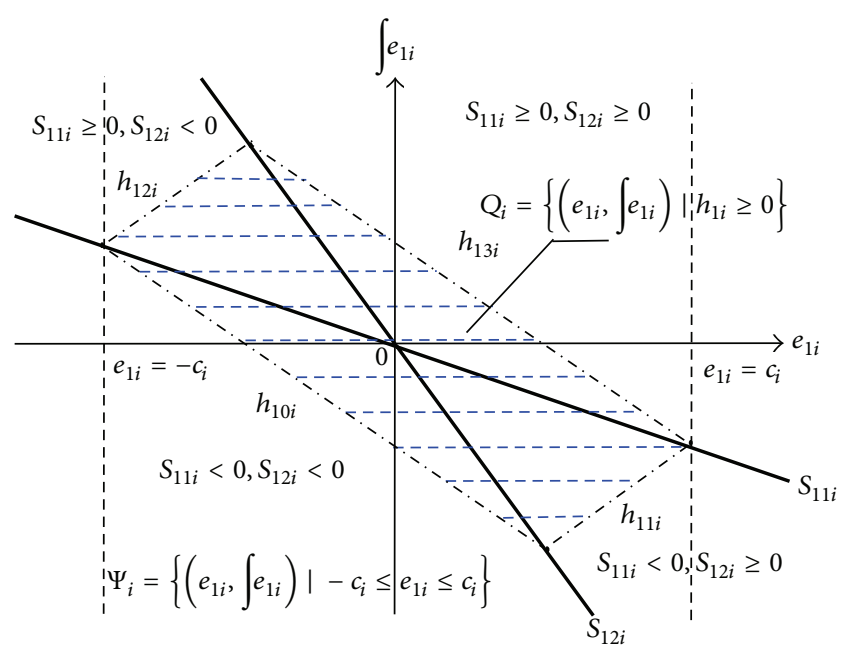

FIgURE 1: The convex set $Q_{i}$ in attitude constraint $\Psi_{i}$.

Step 4. Invoking the coefficients $\xi_{21 i}, \xi_{22 i}$ in (16), the unidirectional auxiliary surface $h_{2 i}$ can be designed as

$$
h_{2 i}=\omega_{2 i 1} \cdot e_{2 i}+\omega_{2 i 2} \cdot \int e_{2 i}+M_{2 i}
$$

where

$$
\begin{aligned}
& \omega_{2 i 1}= \begin{cases}1 & S_{21 i}<0 ; S_{22 i}<0 \\
\omega_{21 i 1} & S_{21 i}<0 ; S_{22 i} \geq 0 \\
\omega_{22 i 1} & S_{21 i} \geq 0 ; S_{22 i}<0 \\
-1 & S_{21 i} \geq 0 ; S_{22 i} \geq 0\end{cases} \\
& \omega_{2 i 2}= \begin{cases}\omega_{20 i 2} & S_{21 i}<0 ; S_{22 i}<0 \\
\omega_{21 i 2} & S_{21 i}<0 ; S_{22 i} \geq 0 \\
\omega_{22 i 2} & S_{21 i} \geq 0 ; S_{22 i}<0 \\
-\omega_{23 i 2} & S_{21 i} \geq 0 ; S_{22 i} \geq 0\end{cases}
\end{aligned}
$$

where $\xi_{22 i}<\omega_{20 i 2}<\xi_{21 i}, \xi_{22 i}<\omega_{23 i 2}<\xi_{21 i}$, and $M_{2 i}>0$ are the designed parameters, and the rest of coefficients can be designed as follows:

$$
\begin{aligned}
& \omega_{21 i 1}=\frac{\left[-\left(\xi_{21 i}+\xi_{22 i}\right)+\left(\omega_{20 i 2}+\omega_{23 i 2}\right)\right]}{\left(\xi_{21 i}-\xi_{22 i}\right)}, \\
& \omega_{21 i 2}=\frac{\left[-2 \xi_{21 i} \xi_{22 i}+\left(\omega_{20 i 2} \xi_{21 i}+\omega_{23 i 2} \xi_{22 i}\right)\right]}{\left(\xi_{21 i}-\xi_{22 i}\right)}, \\
& \omega_{22 i 1}=\frac{\left[\left(\xi_{21 i}+\xi_{22 i}\right)-\left(\omega_{20 i 2}+\omega_{23 i 2}\right)\right]}{\left(\xi_{21 i}-\xi_{22 i}\right)}, \\
& \omega_{21 i 2}=\frac{\left[2 \xi_{21 i} \xi_{22 i}-\left(\omega_{20 i 2} \xi_{21 i}+\omega_{23 i 2} \xi_{22 i}\right)\right]}{\left(\xi_{21 i}-\xi_{22 i}\right)},
\end{aligned}
$$

where $\omega_{21 i 1} \neq 0$ and $\omega_{22 i 1} \neq 0$. Then, the compact form of unidirectional auxiliary surface

$$
\mathbf{h}_{2}=\boldsymbol{\Omega}_{21} \cdot \mathbf{e}_{2}+\boldsymbol{\Omega}_{22} \cdot \int \mathbf{e}_{2}+\mathbf{M}_{2},
$$

where $\mathbf{h}_{2}=\left[h_{21}, h_{22}, h_{23}\right]^{T}, \int \mathbf{e}_{2}=\left[\int e_{21}, \int e_{22}, \int e_{23}\right]^{T}$, $\boldsymbol{\Omega}_{21}=\operatorname{diag}\left\{\omega_{211}, \omega_{221}, \omega_{231}\right\}, \boldsymbol{\Omega}_{22}=\operatorname{diag}\left\{\omega_{212}, \omega_{222}, \omega_{232}\right\}$, and $\mathbf{M}_{2}=\left[M_{21}, M_{22}, M_{23}\right]^{T}$ is a constant vector. Then, there exist the following results for the unidirectional auxiliary surfaces $\mathbf{h}_{1}=\left[h_{11}, h_{12}, h_{13}\right]^{T}$ and $\mathbf{h}_{2}=\left[h_{21}, h_{22}, h_{23}\right]^{T}$.

Lemma 2. For the points $P_{1}(t)=\left(e_{1 i}, \int e_{1 i}\right)$ and $P_{2}(t)=$ $\left(e_{2 i}, \int e_{2 i}\right), i=1,2,3$, it follows that $\delta_{1 i}(t)=M_{1 i}-h_{1 i}\left(P_{1}\right) \geq 0$ and $\delta_{2 i}(t)=M_{2 i}-h_{2 i}\left(P_{2}\right) \geq 0$. And if $\delta_{1 i}(t)=0$, there exists $P_{1}=(0,0)$; if $\delta_{2 i}(t)=0$, there exists $P_{2}=(0,0)$.

Proof. See Appendix A.

Lemma 3. For all $e_{1 i}, e_{2 i} \in R, i=1,2,3$, the functions $\delta_{1 i}(t)$ and $\delta_{2 i}(t)$ in Lemma 2 are continuous functions.

Proof. See Appendix B.

3.2. Robust Adaptive Backstepping Attitude Control Based on Disturbance Observer and Attitude Constraints. In the attitude control design, we combine the backstepping method, adaptive invariant-set-based sliding mode control, and the disturbance observer technique to design a robust adaptive backstepping attitude controller for the AHV system (1) with attitude constraints and propulsive disturbance, and the detailed design process is appended as follows.

\subsubsection{Design Process for the Controller}

Step 1. Invoking (14) and (20), we can define $\boldsymbol{\delta}_{1}$ and $\boldsymbol{\delta}_{2}$ as

$$
\begin{aligned}
& \boldsymbol{\delta}_{1}=\mathbf{M}_{1}-\mathbf{h}_{1}, \\
& \boldsymbol{\delta}_{2}=\mathbf{M}_{2}-\mathbf{h}_{2},
\end{aligned}
$$

where $\boldsymbol{\delta}_{1}=\left[\delta_{11}, \delta_{12}, \delta_{13}\right]^{T}$ and $\boldsymbol{\delta}_{2}=\left[\delta_{21}, \delta_{22}, \delta_{23}\right]^{T}$. Then, the innovative sliding mode surface $\delta_{1 \varepsilon}$ can be defined as

$$
\boldsymbol{\delta}_{1 \varepsilon}=\boldsymbol{\delta}_{1}-\varepsilon_{1} \cdot \operatorname{Sat}\left(\frac{\boldsymbol{\delta}_{1}}{\varepsilon_{1}}\right),
$$

where $\boldsymbol{\delta}_{1 \varepsilon}=\left[\delta_{1 \varepsilon 1}, \delta_{1 \varepsilon 2}, \delta_{1 \varepsilon 3}\right]^{T}, \boldsymbol{\varepsilon}_{1}=\operatorname{diag}\left\{M_{11}, M_{12}, M_{13}\right\}$, $\operatorname{Sat}\left(\boldsymbol{\delta}_{1} / \boldsymbol{\varepsilon}_{1}\right)=\left[\operatorname{Sat}\left(\delta_{11} / M_{11}\right), \operatorname{Sat}\left(\delta_{12} / M_{12}\right), \operatorname{Sat}\left(\delta_{13} / M_{13}\right)\right]^{T}$, and $\operatorname{Sat}(\cdot)$ is the saturation function. From (22), it is clear that

$$
\begin{aligned}
\delta_{1 \varepsilon i} & =\delta_{1 i}-M_{1 i} \cdot \operatorname{Sat}\left(\frac{\delta_{1 i}}{M_{1 i}}\right) \\
& = \begin{cases}0 & \delta_{1 i} \leq M_{1 i} \\
\delta_{1 i}-M_{1 i} & \delta_{1 i}>M_{1 i}\end{cases}
\end{aligned}
$$

Remark 4. It is clear that $\delta_{1 \varepsilon i}=0$ when $\delta_{1 i}=M_{1 i}-h_{1 i} \leq M_{i}$. Considering $Q_{i}=\left\{\left(e_{1 i}, \int e_{1 i}\right) \mid h_{1 i} \geq 0\right\} \subseteq \Psi_{i}$ in (15), we 
can obtain that $\mathbf{y} \in \Psi$ while $\boldsymbol{\delta}_{1 \varepsilon}=0$. Therefore, the attitude constraints will be satisfied when system states are sliding on the sliding surface $\boldsymbol{\delta}_{1 \varepsilon}=0$.

Step 2. In this paper, the nonlinear disturbance observer which is employed to estimate $\mathbf{d}\left(\mathbf{y}, \mathbf{y}_{d}, t\right)$ is defined as

$$
\begin{aligned}
& \widehat{\mathbf{d}}=\mathbf{L}\left(\mathbf{e}_{2}-\boldsymbol{\eta}\right), \\
& \dot{\hat{\boldsymbol{\eta}}}=\mathbf{F}_{f}\left(\mathbf{x}_{1}, \mathbf{x}_{2}\right)+\mathbf{G}_{f}\left(\mathbf{x}_{1}, \mathbf{x}_{2}\right) \cdot \mathbf{u}+\widehat{\mathbf{d}}-\dot{\boldsymbol{v}}_{1}+\mathbf{L}^{-1} \cdot \mathbf{\Omega}_{21}
\end{aligned}
$$

$\cdot \mathbf{E}$,

where $\mathbf{L}=\mathbf{L}^{T}>0$ is a design parameter which should be chosen to render $\mathbf{L}-0.5 \mathbf{I}_{3 \times 3}>0, \mathbf{E}=[1,1,1]^{T}$.

Assumption 5. For the estimation error $\tilde{\mathbf{d}}=\widehat{\mathbf{d}}-\mathbf{d}=$ $\left[\widetilde{d}_{1}, \widetilde{d}_{2}, \widetilde{d}_{3}\right]^{T}$, there exists positive constant $\theta_{i}$ such that $\theta_{i}>$ $-\tau_{1 i} \cdot \widetilde{d}_{i}, i=1,2,3$, where the coefficient $\tau_{1 i}$ is a bounded element in (4).

Step 3. To suppress the estimation error $\widetilde{\mathbf{d}}$ of disturbance, an adaptive item $\widehat{\boldsymbol{\theta}}$ is defined as

$$
\dot{\hat{\boldsymbol{\theta}}}=\lambda \cdot \boldsymbol{\delta}_{1 \varepsilon}
$$

where $\widehat{\boldsymbol{\theta}}=\left[\widehat{\theta}_{1}, \widehat{\theta}_{2}, \widehat{\theta}_{3}\right]^{T}$ and $\lambda>0$ is a design parameter.

Step 4. Defining $\widetilde{\boldsymbol{\theta}}=\widehat{\boldsymbol{\theta}}-\boldsymbol{\theta}$ and $\boldsymbol{\Delta}_{1 \varepsilon}=\operatorname{diag}\left\{\delta_{1 \varepsilon 1}, \delta_{1 \varepsilon 2}, \delta_{1 \varepsilon 3}\right\}$ and considering (7), the virtual control law $\boldsymbol{\nu}_{1}$ is designed as

$$
\begin{aligned}
& \boldsymbol{v}_{1} \\
& =\mathbf{G}_{s}^{-1}\left[-\mathbf{F}_{s}+\dot{\mathbf{y}}_{d}+\boldsymbol{\Omega}_{11}^{-1}\left(-\boldsymbol{\Omega}_{12} \cdot \mathbf{e}_{1}+\mathbf{N}_{1}+\Delta_{1 \varepsilon} \cdot \widehat{\boldsymbol{\theta}}\right)\right],
\end{aligned}
$$

where $\mathbf{N}_{1}=\left[N_{11}, N_{12}, N_{13}\right]^{T}, N_{1 i} \geq 0, i=1,2,3$ are designed approaching laws. Using the output of the designed nonlinear disturbance observer (24) and considering Assumptions 3 and 4, the robust attitude control law is designed as

$$
\begin{aligned}
\mathbf{u}= & \mathbf{G}_{f}^{-1}\left(-\mathbf{F}_{f}-\widehat{\mathbf{d}}+\dot{\boldsymbol{\nu}}_{1}+\mathbf{\Omega}_{21}^{-1}\left(-\boldsymbol{\Omega}_{22} \cdot \mathbf{e}_{2}+\mathbf{N}_{2}\right)\right. \\
& \left.-\boldsymbol{\Omega}_{21}^{-1} \boldsymbol{\Omega}_{11} \mathbf{G}_{s} \mathbf{e}_{2}\right)
\end{aligned}
$$

where $\mathbf{G}_{f}^{-1}$ is the generalized matrix inverse of $\mathbf{G}_{f}, \mathbf{N}_{2}=$ $\left[N_{21}, N_{22}, N_{23}\right]^{T}$, and $N_{2 i}>0.5 \mu_{i}^{2}, i=1,2,3$.

Remark 6. Defining $\mathbf{E}=[1,1,1]^{T}$ and considering $\Delta_{1 \varepsilon}=$ $\operatorname{diag}\left\{\delta_{1 \varepsilon 1}, \delta_{1 \varepsilon 2}, \delta_{1 \varepsilon 3}\right\}$ and $\delta_{1 \varepsilon}=\left[\delta_{1 \varepsilon 1}, \delta_{1 \varepsilon 2}, \delta_{1 \varepsilon 3}\right]^{T}$, we can obtain

$$
\begin{aligned}
& \mathbf{E}^{T} \cdot \Delta_{1 \varepsilon}=\boldsymbol{\delta}_{1 \varepsilon}^{T} ; \\
& \mathbf{E}^{T} \cdot \boldsymbol{\delta}_{1 \varepsilon}=\delta_{1 \varepsilon 1}+\delta_{1 \varepsilon 2}+\delta_{1 \varepsilon 3} .
\end{aligned}
$$

3.2.2. Proof for the Stability of Sliding Mode Surface. The discussion for the stability of sliding mode surface $\boldsymbol{\delta}_{1 \varepsilon}=0$ is given in this part. Considering $\boldsymbol{\delta}_{1 \varepsilon}=0$, the control law in (26) can be rewritten as follows:

$$
\boldsymbol{\nu}_{1}=\mathbf{G}_{s}^{-1}\left(-\mathbf{F}_{s}+\dot{\mathbf{y}}_{d}+\mathbf{\Omega}_{11}^{-1}\left(-\mathbf{\Omega}_{12} \cdot \mathbf{e}_{1}+\mathbf{N}_{1}\right)\right) .
$$

Substituting (29) into (7) yields

$$
\dot{\mathbf{e}}_{1}=\mathbf{\Omega}_{11}^{-1}\left(-\mathbf{\Omega}_{12} \cdot \mathbf{e}_{1}+\mathbf{N}_{1}\right)+\mathbf{G}_{s} \cdot \mathbf{e}_{2} .
$$

Choose the Lyapunov function candidate as

$$
V_{1}=\mathbf{E}^{T} \boldsymbol{\delta}_{1}
$$

From Lemmas 2 and 3, it is clear that the Lyapunov function $V_{1}$ is a positive and continuous function. And there exists $\mathbf{e}_{1}=$ 0 while $V_{1}=0$. Invoking (14) and (21), the time derivative of $V_{1}$ can be written as

$$
\dot{V}_{1}=\mathbf{E}^{T} \dot{\boldsymbol{\delta}}_{1}=\mathbf{E}^{T}\left(-\boldsymbol{\Omega}_{11} \dot{\mathbf{e}}_{1}-\boldsymbol{\Omega}_{12} \mathbf{e}_{1}\right) .
$$

Substituting (30) into (32) yields

$$
\dot{V}_{1}=-\mathbf{E}^{T} \mathbf{N}_{1}-\mathbf{E}^{T} \boldsymbol{\Omega}_{11} \mathbf{G}_{s} \mathbf{e}_{2} .
$$

It is apparent that the first term on the right-hand side of (33) is stable. Furthermore, the second term will be canceled as follows.

Invoking (4), it is noted that the time derivative of propulsive disturbance $\dot{\mathbf{d}}_{1}=0$ while $\boldsymbol{\delta}_{1 \varepsilon}=0$. Then, we can obtain $\dot{\mathbf{d}}=\dot{\mathbf{d}}_{2}=\boldsymbol{\tau}_{2}$ from (2), (3), and (4) and $\boldsymbol{\delta}_{1 \varepsilon}=0$. Considering the definition of $\widetilde{\mathbf{d}}$ and substituting (27) into (8) yield

$$
\dot{\mathbf{e}}_{2}=-\tilde{\mathbf{d}}+\boldsymbol{\Omega}_{21}^{-1}\left(-\boldsymbol{\Omega}_{22} \mathbf{e}_{2}+\mathbf{N}_{2}\right)-\boldsymbol{\Omega}_{21}^{-1} \boldsymbol{\Omega}_{11} \mathbf{G}_{s} \mathbf{e}_{2} .
$$

For considering the stability of all signals for the closed-loop control system, the Lyapunov function candidate is chosen as

$$
V=V_{1}+0.5 \widetilde{\mathbf{d}}^{T} \widetilde{\mathbf{d}}+\mathbf{E}^{T} \boldsymbol{\delta}_{2} .
$$

The time derivative of $V$ is

$$
\dot{V}=\dot{V}_{1}+\widetilde{\mathbf{d}}^{T} \dot{\overrightarrow{\mathbf{d}}}+\mathbf{E}^{T} \dot{\boldsymbol{\delta}}_{2}
$$

Invoking (8), (21), (24), (27), and Assumption 3, the time derivative is

$$
\begin{aligned}
\dot{V} & =-\mathbf{E}^{T} \mathbf{N}_{1}-\mathbf{E}^{T} \mathbf{N}_{2}-\widetilde{\mathbf{d}}^{T} \mathbf{L} \widetilde{\mathbf{d}}-\tilde{\mathbf{d}} \dot{\mathbf{d}} \\
& \leq-\mathbf{E}^{T} \mathbf{N}_{1}-\mathbf{E}^{T} \mathbf{N}_{2}-\widetilde{\mathbf{d}}^{T}\left(\mathbf{L}-0.5 \mathbf{I}_{3 \times 3}\right) \tilde{\mathbf{d}}+0.5 \dot{\mathbf{d}}^{T} \dot{\mathbf{d}} \\
& <-\mathbf{E}^{T} \mathbf{N}_{1}-\mathbf{E}^{T} \mathbf{N}_{2}+0.5 \boldsymbol{\mu}^{T} \boldsymbol{\mu}-\widetilde{\mathbf{d}}^{T}\left(\mathbf{L}-0.5 \mathbf{I}_{3 \times 3}\right) \tilde{\mathbf{d}},
\end{aligned}
$$

where $\boldsymbol{\mu}=\left[\mu_{1}, \mu_{2}, \mu_{3}\right]^{T}$.

Theorem 7. Considering the error systems (7) and (8) with the unknown propulsive disturbance, the disturbance observer is designed as (24) and the robust backstepping attitude control scheme is proposed as (26) and (27). Then, the attitude control error of $A H V$ is convergent when the system states are sliding on the innovative sliding surface $\boldsymbol{\delta}_{1 \varepsilon}=0$ in (22). 
Proof. Invoking (37) and considering $\mathbf{L}-0.5 \mathbf{I}_{3 \times 3}>0, N_{1 i} \geq$ 0 , and $N_{2 i}>0.5 \mu_{i}^{2}, i=1,2,3$, we have

$$
\begin{aligned}
\dot{V} & <-\mathbf{E}^{T} \mathbf{N}_{1}-\mathbf{E}^{T} \mathbf{N}_{2}+0.5 \boldsymbol{\mu}^{T} \boldsymbol{\mu}-\tilde{\mathbf{d}}^{T}\left(\mathbf{L}-0.5 \mathbf{I}_{3 \times 3}\right) \tilde{\mathbf{d}} \\
& =-\widetilde{\mathbf{d}}^{T}\left(\mathbf{L}-0.5 \mathbf{I}_{3 \times 3}\right) \tilde{\mathbf{d}}-\sum\left(N_{1 i}+N_{2 i}-0.5 \mu_{i}^{2}\right) \\
& <0 .
\end{aligned}
$$

Thus, the tracking errors $\mathbf{e}_{1}, \mathbf{e}_{2}$ and the disturbance approximation error $\tilde{\mathbf{d}}$ are convergent while the system states are sliding on the innovative sliding mode surface (22).

3.2.3. Proof for the Reaching Phase for Sliding Mode Surface. The discussion for the reaching phase for sliding surface is given in this part. Choose the Lyapunov function candidate as

$$
V_{1}=\mathbf{E}^{T} \boldsymbol{\delta}_{1 \varepsilon}+\frac{1}{2 \lambda} \widetilde{\boldsymbol{\theta}}^{T} \widetilde{\boldsymbol{\theta}}
$$

From Lemmas 2 and 3, it is clear that the Lyapunov function $V_{1}$ is a positive and continuous function, and there exist $\boldsymbol{\delta}_{1 \varepsilon}=$ 0 while $V_{1}=0$. Assume that $\boldsymbol{\delta}_{1 \varepsilon} \neq 0$ and considering (23), there exists the following equation:

$$
\dot{\boldsymbol{\delta}}_{1 \varepsilon}=\dot{\boldsymbol{\delta}}_{1}=-\boldsymbol{\Omega}_{11} \dot{\mathbf{e}}_{1}-\boldsymbol{\Omega}_{12} \mathbf{e}_{1} .
$$

Substituting (7) and (26) into (40) yields

$$
\dot{\boldsymbol{\delta}}_{1 \varepsilon}=-\boldsymbol{\Omega}_{11} \mathbf{G}_{s} \mathbf{e}_{2}-\mathbf{N}_{1}-\Delta_{1 \varepsilon} \cdot \widehat{\boldsymbol{\theta}} .
$$

Invoking (26) and (39), the time derivative of $V_{1}$ can be written as

$$
\dot{V}_{1}=\mathbf{E}^{T} \dot{\boldsymbol{\delta}}_{1 \varepsilon}+\frac{\boldsymbol{\theta}^{T} \dot{\hat{\boldsymbol{\theta}}}}{\lambda}
$$

Substituting (25), (28), and (40) into (42) yields

$$
\begin{aligned}
\dot{V}_{1} & =\mathbf{E}^{T}\left(-\boldsymbol{\Omega}_{11} \mathbf{G}_{s} \mathbf{e}_{2}-\mathbf{N}_{1}-\Delta_{1 \varepsilon} \cdot \widehat{\boldsymbol{\theta}}\right)+\boldsymbol{\delta}_{1 \varepsilon}^{T} \tilde{\boldsymbol{\theta}} \\
& =\mathbf{E}^{T}\left(-\boldsymbol{\Omega}_{11} \mathbf{G}_{s} \mathbf{e}_{2}-\mathbf{N}_{1}\right)-\boldsymbol{\delta}_{1 \varepsilon}^{T} \cdot \widehat{\boldsymbol{\theta}}+\boldsymbol{\delta}_{1 \varepsilon}^{T} \tilde{\boldsymbol{\theta}} .
\end{aligned}
$$

Invoking (2), (4), and (5) yields

$$
\dot{\mathbf{d}}=\dot{\mathbf{d}}_{1}+\dot{\mathbf{d}}_{2}=\tau_{1} \cdot \boldsymbol{\delta}_{1 \varepsilon}+\tau_{2} .
$$

Considering the stability of all signals for the closed-loop control system, the Lyapunov function candidate is chosen as

$$
V=V_{1}+0.5 \widetilde{\mathbf{d}}^{T} \widetilde{\mathbf{d}}+\mathbf{E}^{T} \boldsymbol{\delta}_{2} .
$$

The time derivative of $V$ is

$$
\dot{V}=\dot{V}_{1}+\widetilde{\mathbf{d}}^{T} \dot{\overrightarrow{\mathbf{d}}}+\mathbf{E}^{T} \dot{\boldsymbol{\delta}}_{2}=\dot{V}_{1}+\widetilde{\mathbf{d}}^{T}(\dot{\overrightarrow{\mathbf{d}}}-\dot{\mathbf{d}})+\mathbf{E}^{T} \dot{\boldsymbol{\delta}}_{2}
$$

Invoking (21), (24), (27), (43), and (44), the time derivative of $V$ is

$$
\begin{aligned}
\dot{V}= & -\mathbf{E}^{T}\left(\mathbf{N}_{1}+\mathbf{N}_{2}\right)-\boldsymbol{\delta}_{1 \varepsilon}^{T} \widehat{\boldsymbol{\theta}}+\boldsymbol{\delta}_{1 \varepsilon}^{T} \widetilde{\boldsymbol{\theta}}+\widetilde{\mathbf{d}}^{T}(-\mathbf{L}) \tilde{\mathbf{d}} \\
& -\widetilde{\mathbf{d}}^{T}\left(\boldsymbol{\tau}_{1} \cdot \boldsymbol{\delta}_{1 \varepsilon}+\boldsymbol{\tau}_{2}\right) \\
\leq & -\mathbf{E}^{T}\left(\mathbf{N}_{1}+\mathbf{N}_{2}\right)-\boldsymbol{\delta}_{1 \varepsilon}^{T} \widehat{\boldsymbol{\theta}}+\boldsymbol{\delta}_{1 \varepsilon}^{T} \widetilde{\boldsymbol{\theta}} \\
& -\widetilde{\mathbf{d}}^{T}\left(\mathbf{L}-0.5 \mathbf{I}_{3 \times 3}\right) \tilde{\mathbf{d}}-\widetilde{\mathbf{d}}^{T} \boldsymbol{\tau}_{1} \boldsymbol{\delta}_{1 \varepsilon}+0.5 \boldsymbol{\tau}_{2}^{T} \boldsymbol{\tau}_{2} .
\end{aligned}
$$

Considering Assumption 5, $\boldsymbol{\delta}_{1 \varepsilon}>0,(22)$, and (23), we have

$$
\begin{aligned}
\dot{V} \leq & -\mathbf{E}^{T}\left(\mathbf{N}_{1}+\mathbf{N}_{2}\right)+\boldsymbol{\delta}_{1 \varepsilon}^{T}\left[-\widehat{\boldsymbol{\theta}}+\widetilde{\boldsymbol{\theta}}-\boldsymbol{\tau}_{1} \widetilde{\mathbf{d}}\right] \\
& -\widetilde{\mathbf{d}}^{T}\left(\mathbf{L}-0.5 \mathbf{I}_{3 \times 3}\right) \tilde{\mathbf{d}}+0.5 \boldsymbol{\tau}_{2}^{T} \boldsymbol{\tau}_{2} \\
\leq & -\mathbf{E}^{T}\left(\mathbf{N}_{1}+\mathbf{N}_{2}\right)+\boldsymbol{\delta}_{1 \varepsilon}^{T}[-\widehat{\boldsymbol{\theta}}+\widetilde{\boldsymbol{\theta}}+\boldsymbol{\theta}] \\
& -\widetilde{\mathbf{d}}^{T}\left(\mathbf{L}-0.5 \mathbf{I}_{3 \times 3}\right) \tilde{\mathbf{d}}+0.5 \boldsymbol{\tau}_{2}^{T} \boldsymbol{\tau}_{2} .
\end{aligned}
$$

Substituting $\widetilde{\boldsymbol{\theta}}=\widehat{\boldsymbol{\theta}}-\boldsymbol{\theta}$ into (48) yields

$$
\dot{V} \leq-\mathbf{E}^{T}\left(\mathbf{N}_{1}+\mathbf{N}_{2}\right)-\widetilde{\mathbf{d}}^{T}\left(\mathbf{L}-0.5 \mathbf{I}_{3 \times 3}\right) \tilde{\mathbf{d}}+0.5 \boldsymbol{\tau}_{2}^{T} \boldsymbol{\tau}_{2}
$$

Theorem 8. Considering the attitude motion dynamics (1) of the AHV with attitude constraints and unknown propulsive disturbance, the disturbance observer is designed as (24) and the robust backstepping attitude control scheme is proposed as (26) and (27). Then, attitude controller error of $A H V$ is convergent under the attitude constraints (3). Meanwhile, the system states will arrive at the sliding surface $\boldsymbol{\delta}_{1 \varepsilon}=0$, and this surface also can be proved as a positively invariant set.

Proof. According to (49), we have

$$
\dot{V} \leq-\widetilde{\mathbf{d}}^{T}\left(\mathbf{L}-0.5 \mathbf{I}_{3 \times 3}\right) \tilde{\mathbf{d}}-\sum\left(N_{1 i}+N_{2 i}-0.5 \mu_{i}^{2}\right) .
$$

Substituting $N_{1 i} \geq 0, N_{2 i}>0.5 \mu_{i}^{2}$, and $\mathbf{L}-0.5 \mathbf{I}_{3 \times 3}>0$ into (50) yields

$$
\dot{V}<0 \text {. }
$$

Thus, the system states will arrive at the sliding surface $\boldsymbol{\delta}_{1 \varepsilon}=$ 0 . From Remark 4, it is noted that attitude constraints (3) will be satisfied when system states are sliding on sliding surface $\boldsymbol{\delta}_{1 \varepsilon}=0$.

Considering the stability of sliding surface as shown in Theorem 8, the attitude control error of AHV is convergent under the attitude constraints (3). From (39), (45), and (51), there exists $\boldsymbol{\delta}_{1 \varepsilon}(t)=0$ for all $t>t_{1}$ if $V\left(t_{1}\right)=0$. And the sliding surface $\delta_{1 \varepsilon}(t)=0$ can be rewritten as a closed set $\mathbf{Q}$ as shown in (15) and Remark 4. Then, the sliding surface $\boldsymbol{\delta}_{1 \varepsilon}=0$ can be seen as a positively invariant set according to Definition 1.

\section{Simulation Results}

In this section, simulation results are given to illustrate the effectiveness of the proposed adaptive UAS-SMC schemes for AHV with attitude constraints. Suppose that the AHV vehicle lies in the cruise flight phase with the velocity $3000 \mathrm{~m} / \mathrm{s}$ and flight altitude $30 \mathrm{~km}$. The initial attitude and attitude angular velocity conditions are chosen as $\alpha_{0}=0 \mathrm{deg}, \beta_{0}=0 \mathrm{deg}, \mu_{0}=$ $0 \mathrm{deg}$, and $p_{0}=q_{0}=r_{0}=0 \mathrm{deg} / \mathrm{s}$. The attitude constraints for the state errors $\mathbf{e}_{1}=\mathbf{y}-\mathbf{y}_{d}=\left[e_{11}, e_{12}, e_{13}\right]^{T}$ are given as

$$
\begin{gathered}
-1 \mathrm{deg} \leq e_{11} \leq 1 \mathrm{deg}, \\
-0.2 \mathrm{deg} \leq e_{12} \leq 0.2 \mathrm{deg}, \\
-1 \mathrm{deg} \leq e_{13} \leq 1 \mathrm{deg} .
\end{gathered}
$$


In the simulation, we assume that the unknown time-varying disturbance moments imposed on the AHV are

$$
\mathbf{d}\left(\mathbf{y}, \mathbf{y}_{d}, t\right)=\mathbf{d}_{1}\left(\mathbf{y}, \mathbf{y}_{d}, t\right)+\mathbf{d}_{2}(t)
$$

where $\mathbf{d}_{1}=10^{6} \operatorname{diag}\left\{g_{p}^{l}, g_{q}^{m}, g_{r}^{n}\right\} \mathbf{d}_{1 m} ; \mathbf{d}_{2}=10^{6} \operatorname{diag}\left\{g_{p}^{l}, g_{q}^{m}\right.$, $\left.g_{r}^{n}\right\} \mathbf{d}_{2 m} ; \dot{\mathbf{d}}_{1 m}=\sin (20 \alpha) \cdot \boldsymbol{\delta}_{1 \varepsilon} ; \mathbf{d}_{2 m}=[0.2 \sin (\pi t), 3 \sin (\pi t)$, $\sin (\pi t)]^{T}$, and the coefficients $g_{p}^{l}, g_{q}^{m}$, and $g_{r}^{n}$ can be found in [24].

According to the design steps in Section 3, the adaptive UAS-SMC control design parameters are chosen as $\xi_{11}=\operatorname{diag}\{5,5,5\}, \xi_{12}=\operatorname{diag}\{1,1,1\}, \boldsymbol{\xi}_{21}=\operatorname{diag}\{5,5,5\}$, $\boldsymbol{\xi}_{22}=\operatorname{diag}\{2,2,2\}, \mathbf{L}=\operatorname{diag}\{2,2,2\}, \lambda=1$, and $\boldsymbol{\varepsilon}_{1}=$ $\operatorname{diag}\{0.02,0.02,0.02\} ; \omega_{10 i 2}=\omega_{13 i 2}=5 / 3$ and $\omega_{20 i 2}=\omega_{23 i 2}=$ $3, i=1,2,3$. The detailed approaching laws $\mathbf{N}_{1}>0, \mathbf{N}_{2}>0$ can be found in [25], and the innovative sliding mode surface $\boldsymbol{\delta}_{1 \varepsilon}$ can be defined as

$$
\begin{aligned}
\boldsymbol{\delta}_{1 \varepsilon} & =\boldsymbol{\delta}_{1}-\boldsymbol{\varepsilon}_{1} \cdot \operatorname{Sat}\left(\frac{\boldsymbol{\delta}_{1}}{\boldsymbol{\varepsilon}_{1}}\right), \\
\dot{\hat{\boldsymbol{\theta}}} & =\lambda \cdot \boldsymbol{\delta}_{1 \varepsilon} .
\end{aligned}
$$

Then, the simulation results are given as in Figures 2-5.

The attitude and state error responses are shown in Figures 2-5 under SMC and adaptive UAS-SMC methods, respectively. From Figure 2, attack angle $\alpha$ in system (1) is unstable when propulsive disturbance $\mathbf{d}_{1}$ is not considered in the design process of SMC controller. To handle this problem, the conservative parameters and nonlinear disturbance observer are given in the SMC control design as shown in Figure 3. However, the undesirable overshoots are often found with conservative parameters since attitude constraints have not been considered in the design process of SMC scheme. And these overshoots are harmful for the AHV system because they might be out of the attitude constraints as shown in Figure 3. From the state error responses in Figure 4, the attitude constraints can be satisfied with adaptive UAS-SMC method. And harmful overshoots will be removed by the designed positively invariant sets. Therefore, we know that the proposed adaptive UAS-SMC control scheme can efficiently track the desired trajectories with attitude constraints and propulsive disturbance. Figure 4 shows the disturbance estimation under NDO [3] and UAS-NDO with the same coefficient L. Unlike the previous NDO, the switching matrix $\Omega_{21}$ of UAS is introduced in the UAS-NDO. This matrix will improve the performance of disturbance observer (24). Then, we can obtain a new fast-nonlinear disturbance observer for the time-varying propulsive disturbance of AHV systems.

\section{Conclusion}

In this paper, the adaptive UAS-SMC controller with nonlinear disturbance observer has been proposed for the AHV with the unknown propulsive disturbance and the attitude constraints. To handle the propulsive disturbance, a developed fast-nonlinear disturbance observer is proposed to estimate the propulsive disturbance using adaptive method. And an innovative sliding surface is firstly proposed for

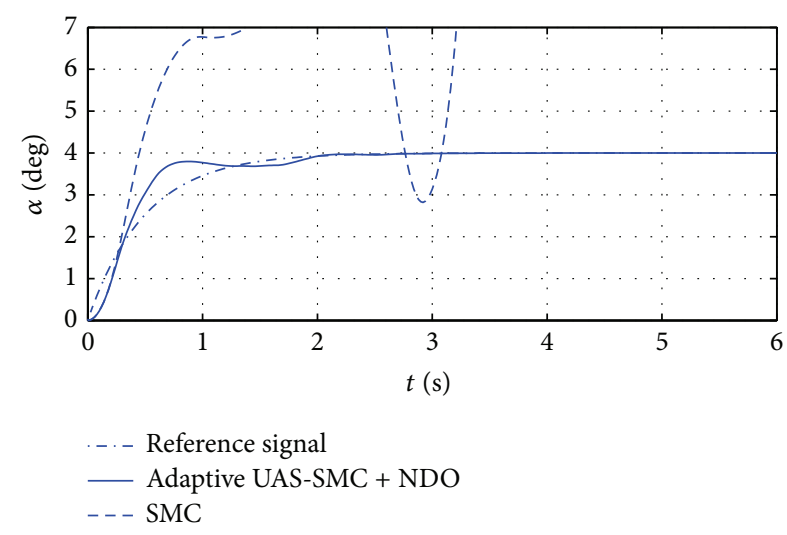

FIgURE 2: The attitude responses of AHV system under SMC and UAS-SMC methods.

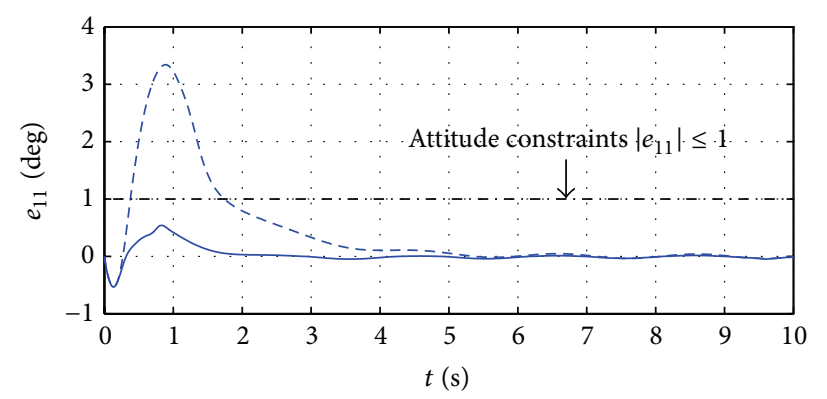

- - - SMC-NDO

— Adaptive UAS-SMC + NDO

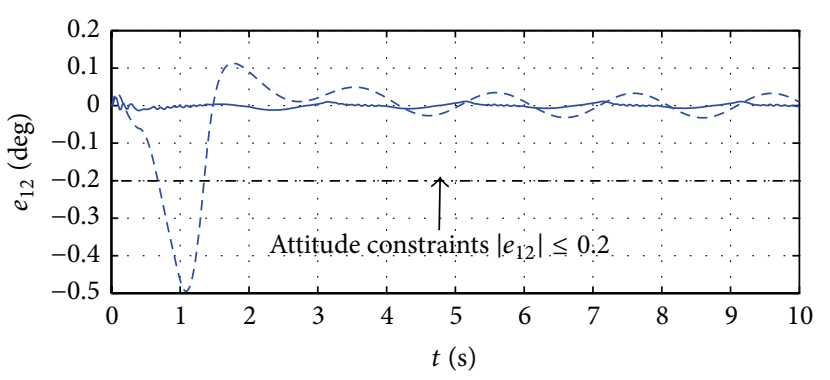

- - - SMC-NDO

- Adaptive UAS-SMC + NDO

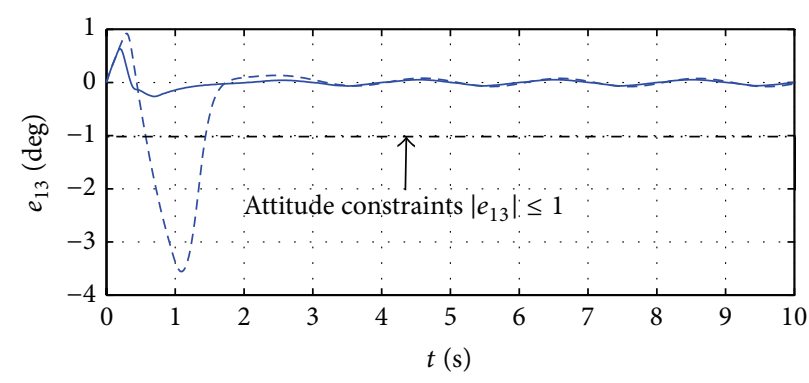

- - SMC-NDO

- Adaptive UAS-SMC + NDO

FIGURE 3: The state error responses under SMC + NDO and adaptive UAS-SMC + NDO 

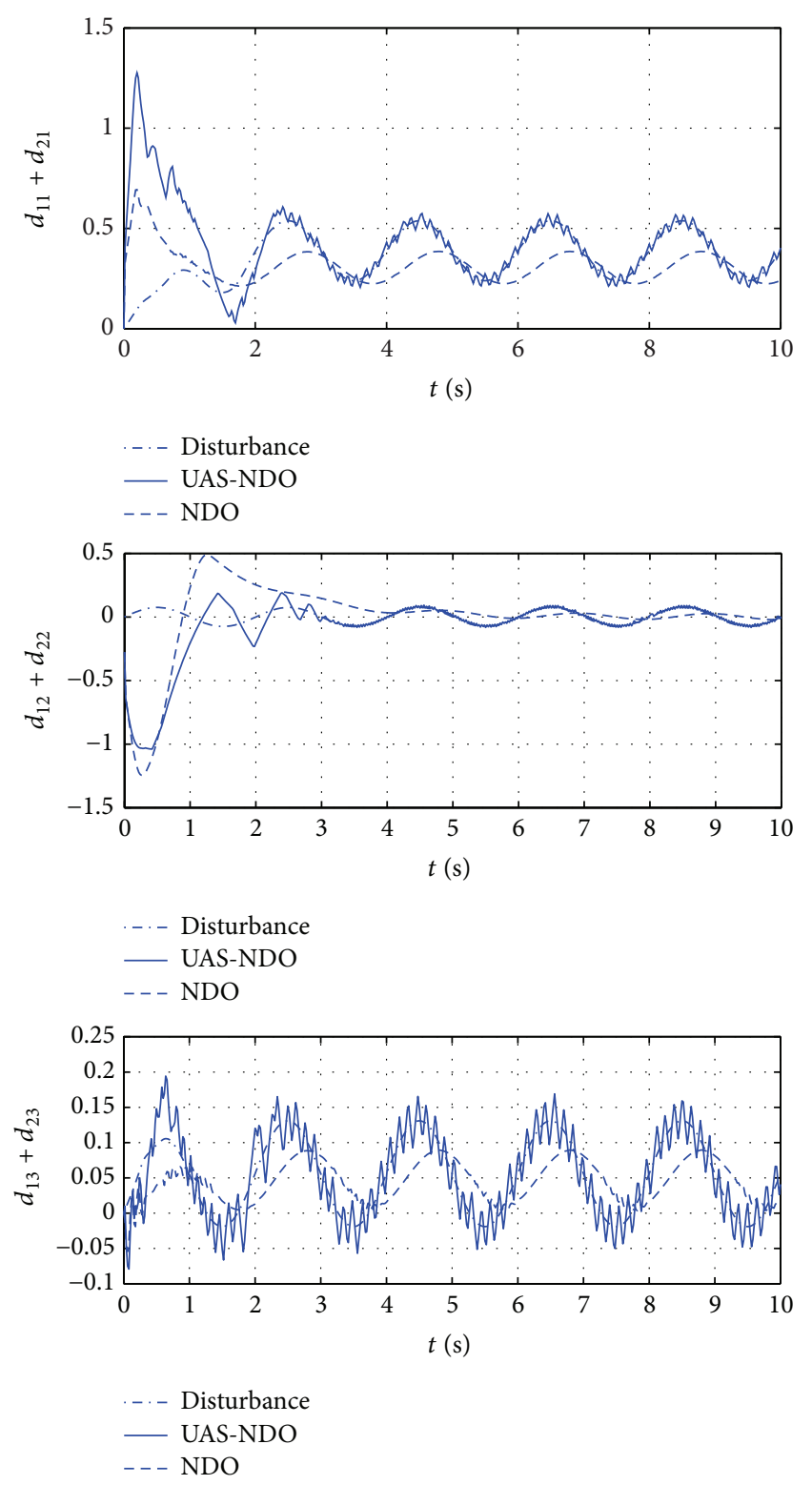

FIgURE 4: The disturbance estimations under NDO [3] and UASNDO with the same coefficient $\mathbf{L}$.

the attitude constraints with invariant set theory. Rigorous analysis has been given for the convergence of all closedloop signals under the proposed control schemes. Simulation results show the effectiveness of the robust adaptive UASSMC scheme for the AHV. In the following study, the robust attitude control scheme can be further developed for the AHV with time-varying attitude constraints.

\section{Appendices}

\section{A. Proof of Lemma 2}

Take the point $P_{1}(t)=\left(e_{1 i}, \int e_{1 i}\right)$ and $\delta_{1 i}(t)=M_{1 i}-h_{1 i}\left(P_{1}\right)$ as examples; we have the following discussions.
From the definition of $h_{1 i}$ in (11), the function $\delta_{1 i}(t)$ is switching among the following subspaces:

$$
\begin{aligned}
& \text { No. } 0_{1 i} \text { Subspace: } S_{11 i}<0, S_{12 i}<0, \\
& \text { No. } 1_{1 i} \text { Subspace: } S_{11 i}<0, S_{12 i} \geq 0 \text {, } \\
& \text { No. } 2_{1 i} \text { Subspace: } S_{11 i} \geq 0, S_{12 i}<0, \\
& \text { No. } 3_{1 i} \text { Subspace: } S_{11 i} \geq 0, S_{12 i} \geq 0 .
\end{aligned}
$$

Since the proofs for the other subspaces are similar, we just prove the conclusions in No. $2_{1 i}$, No. $3_{1 i}$ in this lemma.

Invoking (11) and (13), the function $\delta_{1 i}(t)$ can be written as the following equation for every point $\left(e_{1 i}, \int e_{1 i}\right)$ in No. $2_{i}$ Subspace,

$$
\begin{aligned}
& \delta_{1 i}(t)=M_{1 i}-h_{1 i}=-\omega_{12 i 1} \cdot e_{1 i}-\omega_{12 i 2} \cdot \int e_{1 i} \\
& =-\frac{1}{\xi_{11 i}-\xi_{12 i}}\left[\left(\xi_{12 i}-\omega_{13 i 2}\right)\left(e_{1 i}+\xi_{1 i} \int e_{1 i}\right)\right. \\
& \left.+\left(\xi_{11 i}-\omega_{10 i 2}\right)\left(e_{1 i}+\xi_{2 i} \int e_{1 i}\right)\right] .
\end{aligned}
$$

Since the point $\left(e_{1 i}, \int e_{1 i}\right)$ is located in No. $2_{1 i}$ Subspace, there exists

$$
\begin{aligned}
& S_{11 i}=e_{1 i}+\xi_{11 i} \int e_{1 i} \geq 0, \\
& S_{12 i}=e_{1 i}+\xi_{12 i} \int e_{1 i}<0 .
\end{aligned}
$$

From (10) and (11), it is noted that $\xi_{11 i}>\xi_{12 i}>0, \xi_{12 i}<$ $\omega_{10 i 2}<\xi_{11 i}$, and $\xi_{12 i}<\omega_{13 i 2}<\xi_{11 i}$. Then, we can obtain that $\delta_{1 i}(t)=M_{1 i}-h_{1 i}\left(P_{1}\right)>0$ for every point $\left(e_{1 i}, \int e_{1 i}\right)$ in No. $2_{1 i}$ Subspace.

Invoking (11) and (13), the function $\delta_{1 i}(t)$ can be written as the following equation for every point $\left(e_{1 i}, \int e_{1 i}\right)$ in No. $3_{i}$ Subspace:

$$
\delta_{1 i}(t)=M_{1 i}-h_{1 i}=e_{1 i}+\omega_{13 i 2} \cdot \int e_{1 i} .
$$

Since the point $\left(e_{1 i}, \int e_{1 i}\right)$ is located in No. $3_{1 i}$ Subspace, there exists

$$
\begin{aligned}
& S_{11 i}=e_{1 i}+\xi_{11 i} \int e_{1 i} \geq 0, \\
& S_{12 i}=e_{1 i}+\xi_{12 i} \int e_{1 i} \geq 0 .
\end{aligned}
$$

From (10) and (11), it is noted that $\xi_{11 i}>\xi_{12 i}>0$ and $\xi_{12 i}<$ $\omega_{13 i 2}<\xi_{11 i}$. Then, the coefficient $\omega_{13 i 2}$ can be expressed as

$$
\omega_{13 i 2}=\xi_{12 i}+\tau_{1 i}\left(\xi_{11 i}-\xi_{12 i}\right),
$$

where $0<\tau_{1 i}<1$. Substituting (A.6) into (A.4) and considering (A.5) yield

$$
\begin{aligned}
\delta_{1 i}(t)= & \left(1-\tau_{1 i}\right)\left(e_{1 i}+\xi_{12 i} \int e_{1 i}\right) \\
& +\tau_{1 i}\left(e_{1 i}+\xi_{11 i} \int e_{1 i}\right) \geq 0 .
\end{aligned}
$$



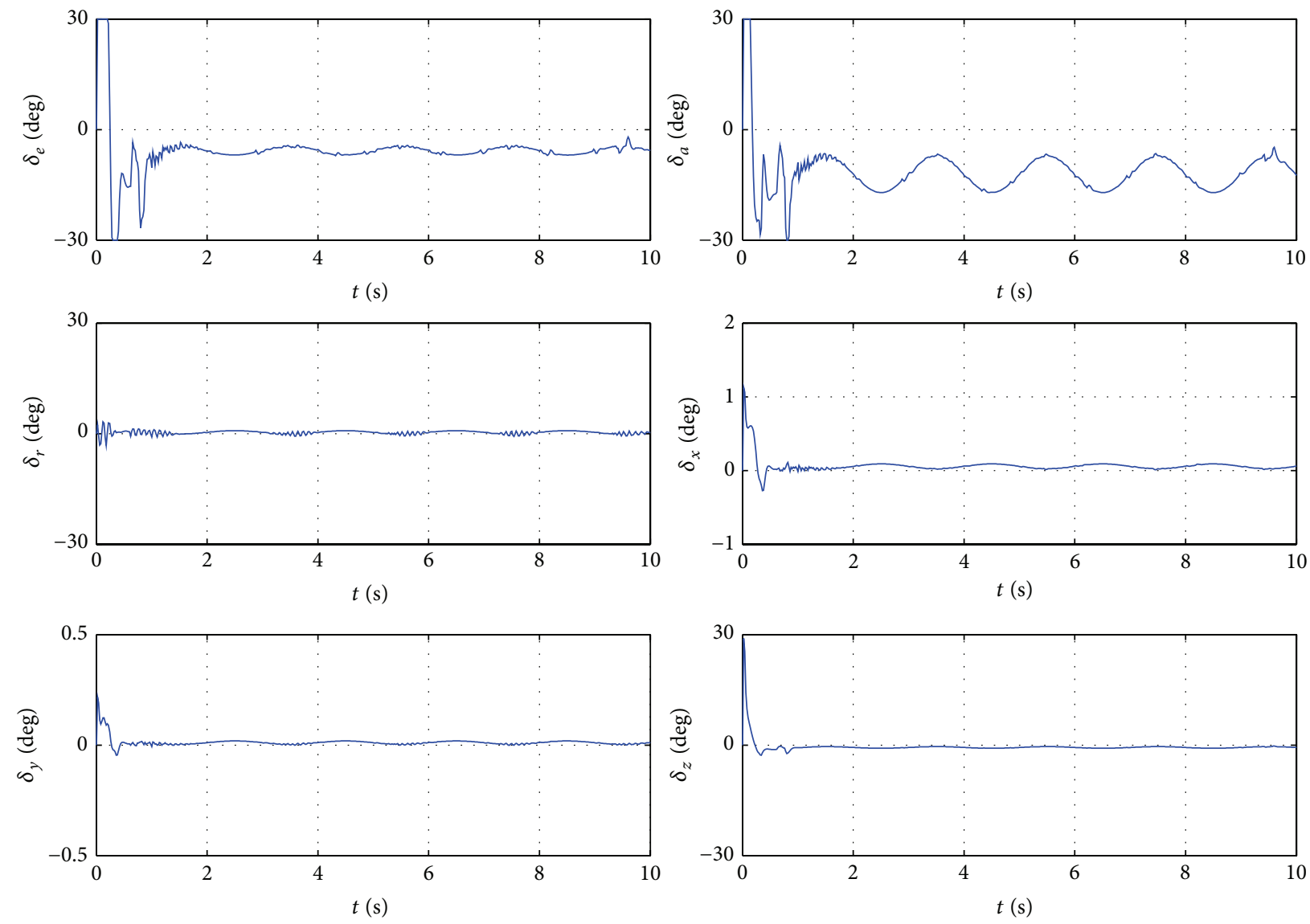

Figure 5: Adaptive UAS-SMC + NDO attitude control input.

Thus, there exists $\delta_{1 i}(t) \geq 0$ for every point $\left(e_{1 i}, \int e_{1 i}\right)$ in No. $3_{1 i}$ Subspace.

Similarly, we have $\delta_{1 i}(t)>0$ for every point $\left(e_{1 i}, \int e_{1 i}\right)$ in No. $1_{1 i}$ and $2_{1 i}$ Subspace. According to above discussion, there exists $\delta_{1 i}(t) \geq 0$ for every $\left(e_{1 i}, \int e_{1 i}\right), e_{1 i} \in R, \int e_{1 i} \in R$. And the function $\delta_{1 i}(t)$ will satisfy the following equation:

$$
\begin{aligned}
& \text { No. } 0_{1 i} \text { Subspace: } \delta_{1 i}(t)>0, \\
& \text { No. } 1_{1 i} \text { Subspace: } \delta_{1 i}(t)>0, \\
& \text { No. } 2_{1 i} \text { Subspace: } \delta_{1 i}(t)>0, \\
& \text { No. } 3_{1 i} \text { Subspace: } \delta_{1 i}(t) \geq 0 .
\end{aligned}
$$

When $\delta_{1 i}(t)=0$, it is clear that the point $\left(e_{1 i}, \int e_{1 i}\right)$ is located in No. $3_{i}$ Subspace. Substituting $\delta_{1 i}(t)=0$ into (A.7) and considering $0<\tau_{1 i}<1, S_{11 i}=e_{1 i}+\xi_{11 i} \int e_{1 i} \geq 0$, and $S_{12 i}=$ $e_{1 i}+\xi_{12 i} \int e_{1 i} \geq 0$ yield

$$
\begin{aligned}
& e_{1 i}+\xi_{11 i} \int e_{1 i}=0, \\
& e_{1 i}+\xi_{12 i} \int e_{1 i}=0 .
\end{aligned}
$$

Considering $\xi_{11 i} \neq \xi_{12 i}$, we have $e_{1 i}=0, \int e_{1 i}=0$. Hence, if $\delta_{1 i}(t)=0$, there exists $P_{1}=\left(e_{1 i}, \int e_{1 i}\right)=(0,0)$. And we can also obtain the similar conclusion for $P_{2}=\left(e_{2 i}, \int e_{2 i}\right)$ and $\delta_{2 i}(t) \geq 0$.

\section{B. Proof of Lemma 3}

Take $\delta_{1 i}(t)=M_{1 i}-h_{1 i}\left(P_{1}\right)$ as an example; we have the following discussions.

Invoking (11) and (13), the function $\delta_{1 i}(t)$ can be written as

$$
\delta_{1 i}(t)=-\omega_{1 i 1} \cdot e_{1 i}-\omega_{1 i 2} \cdot \int e_{1 i}
$$

where

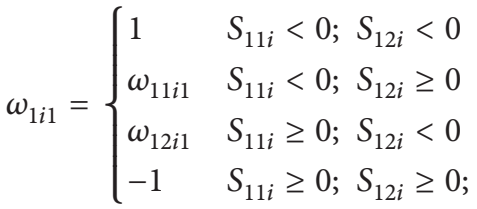

$$
\begin{aligned}
& \omega_{1 i 2}= \begin{cases}\omega_{10 i 2} & S_{11 i}<0 ; S_{12 i}<0 \\
\omega_{11 i 2} & S_{11 i}<0 ; S_{12 i} \geq 0 \\
\omega_{12 i 2} & S_{11 i} \geq 0 ; S_{12 i}<0 \\
-\omega_{13 i 2} & S_{11 i} \geq 0 ; S_{12 i} \geq 0,\end{cases}
\end{aligned}
$$




$$
\begin{aligned}
& \omega_{11 i 1}=\frac{\left[-\left(\xi_{11 i}+\xi_{12 i}\right)+\left(\omega_{10 i 2}+\omega_{13 i 2}\right)\right]}{\left(\xi_{11 i}-\xi_{12 i}\right)}, \\
& \omega_{11 i 2}=\frac{\left[-2 \xi_{11 i} \xi_{12 i}+\left(\omega_{10 i 2} \xi_{11 i}+\omega_{13 i 2} \xi_{12 i}\right)\right]}{\left(\xi_{11 i}-\xi_{12 i}\right)}, \\
& \omega_{12 i 1}=\frac{\left[\left(\xi_{11 i}+\xi_{12 i}\right)-\left(\omega_{10 i 2}+\omega_{13 i 2}\right)\right]}{\left(\xi_{11 i}-\xi_{12 i}\right)}, \\
& \omega_{12 i 2}=\frac{\left[2 \xi_{11 i} \xi_{12 i}-\left(\omega_{13 i 2} \xi_{11 i}+\omega_{10 i 2} \xi_{12 i}\right)\right]}{\left(\xi_{11 i}-\xi_{12 i}\right)} .
\end{aligned}
$$

From the definition of $\omega_{1 i 1}, \omega_{1 i 2}$, function $\delta_{1 i}$ is switched on switching surfaces $S_{11 i}=0$ and $S_{12 i}=0$. Then, the discussion for the continuity of function $\delta_{1 i}$ can be given as follows.

For every point $\left(e_{1 i}, \int e_{1 i}\right)$ on switching surface $S_{11 i}=0$, (10) yields

$$
e_{1 i}=-\xi_{11 i} \int e_{1 i}
$$

Assuming $S_{12 i}<0$ and considering the definition of $\omega_{1 i 1}, \omega_{1 i 2}$, there exists (B.4) for $S_{11 i}=0^{+} \geq 0$ and $S_{12 i}<0$ :

$$
\begin{aligned}
\delta_{1 i}^{+}= & -\omega_{12 i 1} \cdot e_{1 i}-\omega_{12 i 2} \cdot \int e_{1 i} \\
= & -\frac{\left(\xi_{11 i}+\xi_{12 i}\right)-\left(\omega_{10 i 2}+\omega_{13 i 2}\right)}{\xi_{11 i}-\xi_{12 i}} \cdot e_{1 i} \\
& -\frac{2 \xi_{11 i} \xi_{12 i}-\left(\omega_{13 i 2} \xi_{11 i}+\omega_{10 i 2} \xi_{12 i}\right)}{\xi_{11 i}-\xi_{12 i}} \cdot \int e_{1 i} .
\end{aligned}
$$

Substituting (B.3) into (B.4) yields

$$
\delta_{1 i}^{+}=\left(\xi_{11 i}-\omega_{10 i 2}\right) \int e_{1 i} .
$$

Assuming $S_{12 i}<0$ and considering the definition of $\omega_{1 i 1}, \omega_{1 i 2}$, there exists (B.6) for $S_{11 i}=0^{-}<0$ and $S_{12 i}<0$ :

$$
\delta_{1 i}^{-}=-e_{1 i}-\omega_{10 i 2} \cdot \int e_{1 i} .
$$

Substituting (B.3) into (B.6) yields

$$
\delta_{1 i}^{-}=\left(\xi_{11 i}-\omega_{10 i 2}\right) \int e_{1 i} .
$$

Since $\delta_{1 i}^{+}=\delta_{1 i}^{-}$, function $\delta_{1 i}$ is continuous on switching surface $S_{11 i}=0$ while $S_{12 i}<0$. Similarly, function $\delta_{1 i}$ is continuous on switching surface $S_{11 i}=0$ while $S_{12 i} \geq 0$. Hence, $\delta_{1 i}$ is a continuous function for every point $\left(e_{1 i}, \int e_{1 i}\right)$ on switching surface $S_{11 i}=0$. And we can also have the same result for every point $\left(e_{1 i}, \int e_{1 i}\right)$ on switching surface $S_{12 i}=0$.

From the above discussion, function $\delta_{1 i}(t)$ is continuous on switching $S_{11 i}=0, S_{12 i}=0$. Then, we can obtain that the function $\delta_{1 i}(t)$ in Lemma 2 is a continuous function for all $e_{1 i} \in R$. Meanwhile, we can obtain the same conclusion for $\delta_{2 i}(t)$ in Lemma 2.

\section{Conflict of Interests}

The authors declare that there is no conflict of interests regarding the publication of this paper.

\section{Acknowledgments}

This work was partially supported by National Natural Science Foundation (NNSF) of China under Grants 61374212, 61174102, 61304099, and 11402117; Postdoctoral Fund of Jiangsu Province 1401023; Pre-Research Foundation of General Equipment Department 9140C300305140C30140.

\section{References}

[1] B. Jiang, Z. Gao, P. Shi, and Y. Xu, "Adaptive fault-tolerant tracking control of near-space vehicle using Takagi-Sugeno fuzzy models," IEEE Transactions on Fuzzy Systems, vol. 18, no. 5, pp. 1000-1007, 2010.

[2] B. Jiang, Y. Xu, P. Shi, and Z. Gao, "Robust fault-tolerant tracking control for a near-space vehicle using a sliding mode approach," Proceedings of the Institution of Mechanical Engineers. Part I: Journal of Systems and Control Engineering, vol. 225, no. 8, pp. 1173-1184, 2011.

[3] Y. Du, Q. Wu, C. Jiang, and J. Wen, "Adaptive functional link network control of near-space vehicles with dynamical uncertainties," Journal of Systems Engineering and Electronics, vol. 21, no. 5, pp. 868-876, 2010.

[4] D. Hou, Q. Wang, and C. Dong, "Output feedback dynamic surface controller design for airbreathing hypersonic flight vehicle," Automatica Sinica, vol. 2, no. 2, pp. 186-197, 2015.

[5] J. Yang, Z. Zhao, S. Li, and W. X. Zheng, "Nonlinear disturbance observer enhanced predictive control for airbreathing hypersonic vehicles," in Proceedings of the 33rd Chinese Control Conference (CCC '14), pp. 3668-3673, Nanjing, China, July 2014.

[6] H. Sun, S. Li, and C. Sun, "Adaptive fault-tolerant controller design for airbreathing hypersonic vehicle with input saturation," Journal of Systems Engineering and Electronics, vol. 24, no. 3, pp. 488-499, 2013.

[7] J. Yang, S. Li, C. Sun, and L. Guo, "Nonlinear-disturbanceobserver-based robust flight control for airbreathing hypersonic vehicles," IEEE Transactions on Aerospace and Electronic Systems, vol. 49, no. 2, pp. 488-499, 2013.

[8] Z.-Y. Liu, H.-N. Wu, and L. Guo, "Disturbance-observerbased guaranteed cost fuzzy control for airbreathing hypersonic vehicles," in Proceedings of the 32nd Chinese Control Conference (CCC '13), pp. 3476-3481, Xi'an, China, July 2013.

[9] H. Sun, S. Li, Y. Jun, and G. Lei, "Nonlinear disturbance observer-based backstepping control for airbrathing hypersonic vehciles with mismatched disturbances," Control Theory \& Applications, vol. 8, no. 17, pp. 1852-1865, 2014.

[10] J.-T. Chang, D.-R. Yu, and W. Bao, "Characteristic analysis of unstart/restart of hypersonic inlets caused by variations of attack angle of freestream," Journal of Aerospace Power, vol. 23, no. 5, pp. 816-821, 2008.

[11] Z. Pu, X. Tan, G. Fan, and J. Yi, "Uncertainty analysis and robust trajectory linearization control of a flexible air-breathing hypersonic vehicle," Acta Astronautica, vol. 101, no. 1, pp. 16-32, 2014.

[12] H. Li, Z. Li, S. Xue, and D. Xu, "Composite control design with sliding mode for a hypersonic aircraft," in Proceedings of the 3 rd 
International Symposium on Systems and Control in Aeronautics and Astronautics (ISSCAA '10), pp. 1473-1478, IEEE, Harbin, China, June 2010.

[13] J. Zhao, B. Jiang, P. Shi, Z. Gao, and D. Xu, "Fault-tolerant control design for near-space vehicles based on a dynamic terminal sliding mode technique," Proceedings of the Institution of Mechanical Engineers. Part I: Journal of Systems and Control Engineering, vol. 226, no. 6, pp. 787-794, 2012.

[14] P. Wang, L. Liu, J. Wu, and G. Tang, "Attitude control for near space vehicle based on new terminal sliding mode control," in Proceedings of the 31st Chinese Control Conference (CCC '12), pp. 547-551, Hefei, China, July 2012.

[15] J. Yin and Y. Du, "Sliding mode attitude control with input constraints for near space vehicles in reentry phase," in Proceedings of the 32nd Chinese Control Conference (CCC '13), pp. 10641069, Xian, China, July 2013.

[16] J. Zhao, B. Jiang, P. Shi, and H. Liu, "Adaptive dynamic sliding mode control for near space vehicles under actuator faults," Circuits, Systems, and Signal Processing, vol. 32, no. 5, pp. 22812296, 2013.

[17] J. Wang, Q. Zong, R. Su, and B. Tian, "Continuous high order sliding mode controller design for a flexible air-breathing hypersonic vehicle," ISA Transactions, vol. 53, no. 3, pp. 690698, 2014.

[18] J. Li, B. Zuo, M. Duan, and J. Zhang, "Adaptive Terminal sliding mode control for air-breathing hypersonic vehicles under control input constraints," Acta Aeronautica et Astronautica Sinica, vol. 33, no. 2, pp. 220-233, 2012.

[19] Q. Zong, J. Wang, and Y. Tao, "Adaptive high-order dynamic sliding mode control for a flexible air-breathing hypersonic vehicle," International Journal of Robust and Nonlinear Control, vol. 23, no. 15, pp. 1718-1736, 2013.

[20] X. Hu, L. Wu, C. Hu, and H. Gao, "Adaptive sliding mode tracking control for a flexible air-breathing hypersonic vehicle," Journal of the Franklin Institute, vol. 349, no. 2, pp. 559-577, 2012.

[21] Q. Zong, Q. Dong, F. Wang, and B. Tian, "Super twisting sliding mode control for a flexible air-breathing hypersonic vehicle based on disturbance observer," Information Sciences, vol. 58, no. 7, pp. 1-15, 2015.

[22] L. Cheng, C. S. Jiang, and M. Pu, "Online-SVR-compensated nonlinear generalized predictive control for hypersonic vehicles," Science China Information Sciences, vol. 54, no. 3, pp. 551562, 2011.

[23] Z. F. Gao, B. Jiang, R. Y. Qi, and Y. Xu, "Robust reliable control for a near space vehicle with parametric uncertainties and actuator faults," International Journal of Systems Science, vol. 42, no. 12, pp. 2113-2124, 2011.

[24] Y. L. Du, Q. X. Wu, C. S. Jiang, and J. Wen, "Adaptive functional link network control of near-space vehicles with dynamical uncertainties," Journal of Systems Engineering and Electronics, vol. 21, no. 5, pp. 868-876, 2010.

[25] J. Fu, Q.-X. Wu, W.-H. Chen, and X.-G. Yan, "Chatteringfree condition for sliding mode control with unidirectional auxiliary surfaces," Transactions of the Institute of Measurement and Control, vol. 35, no. 5, pp. 593-605, 2013. 


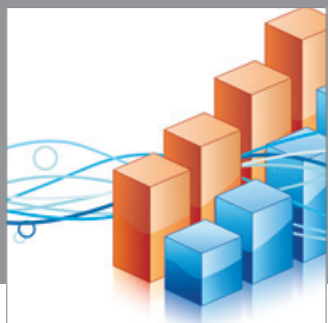

Advances in

Operations Research

mansans

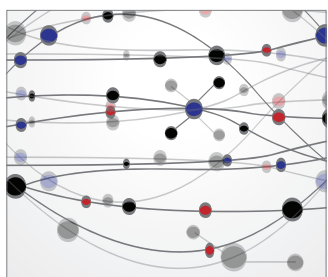

The Scientific World Journal
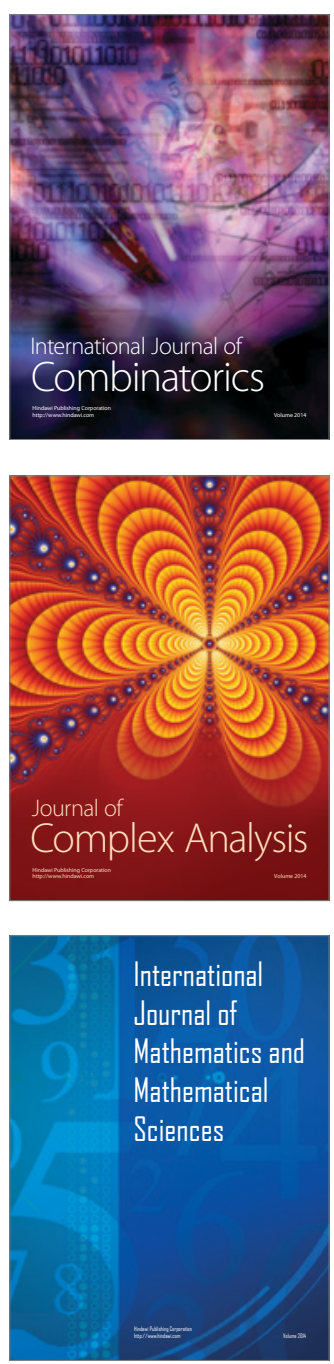
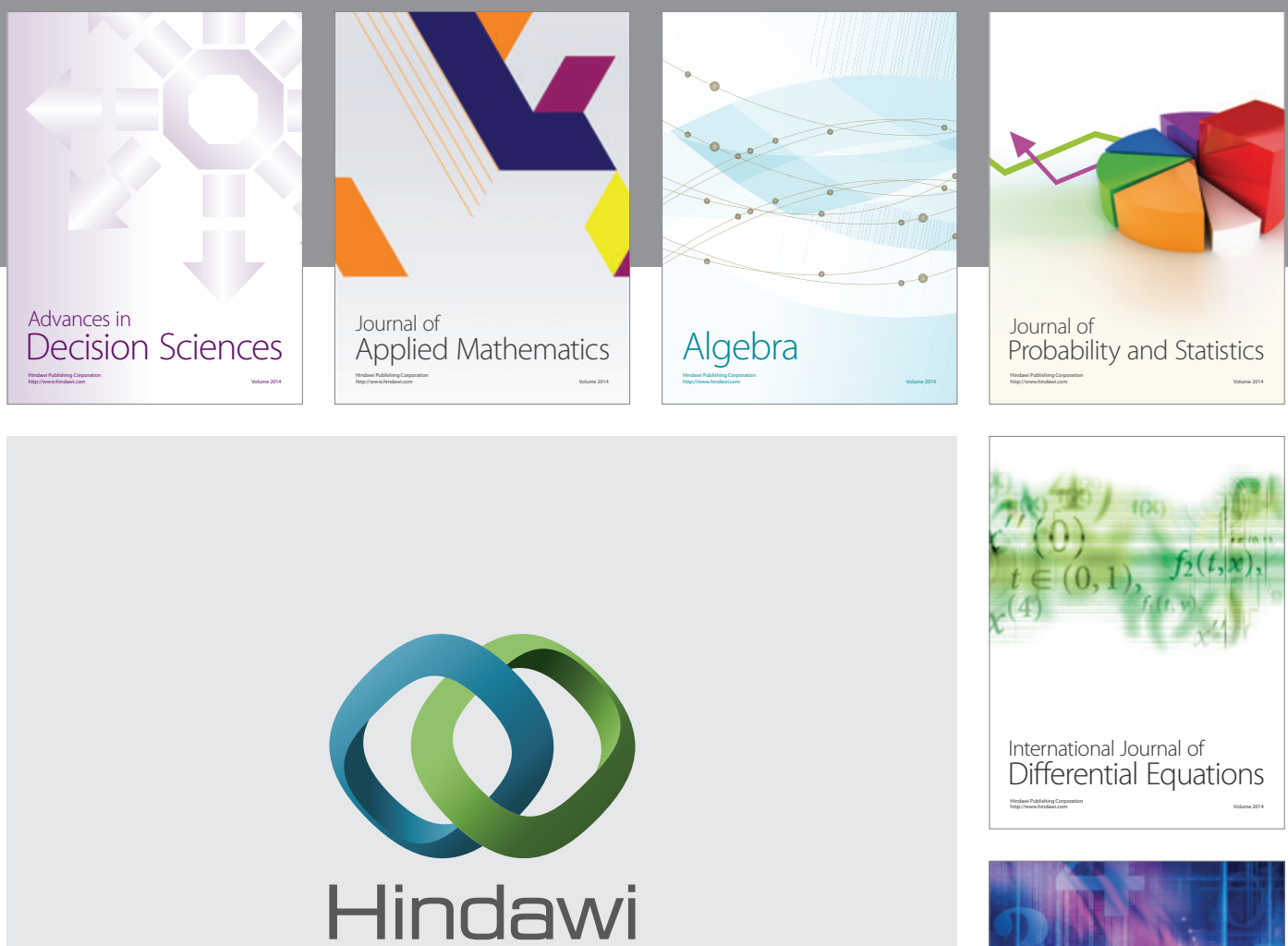

Submit your manuscripts at http://www.hindawi.com
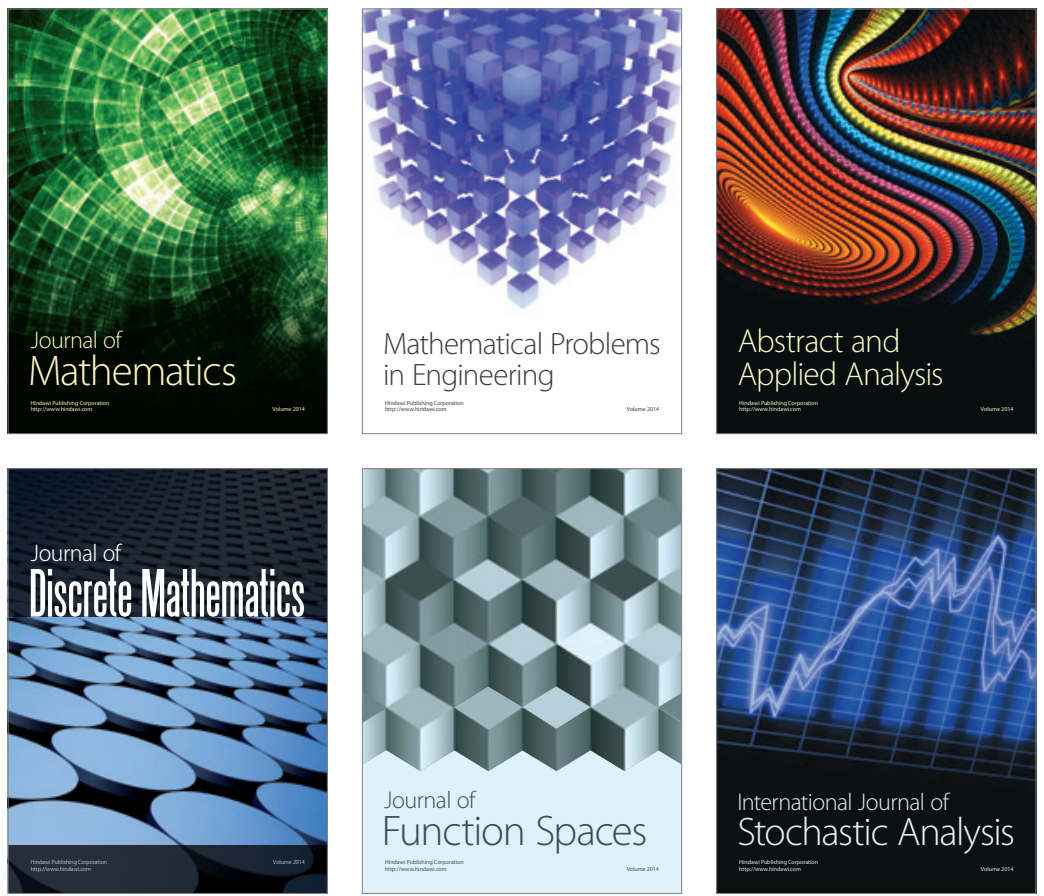

Journal of

Function Spaces

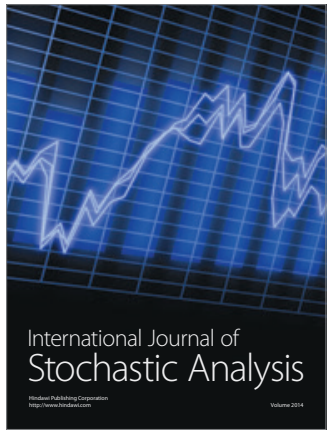

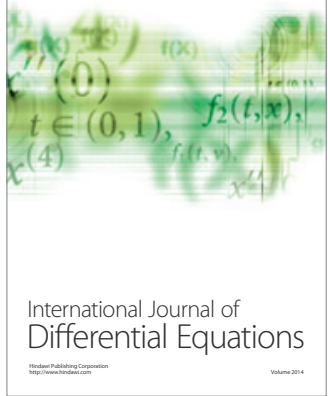
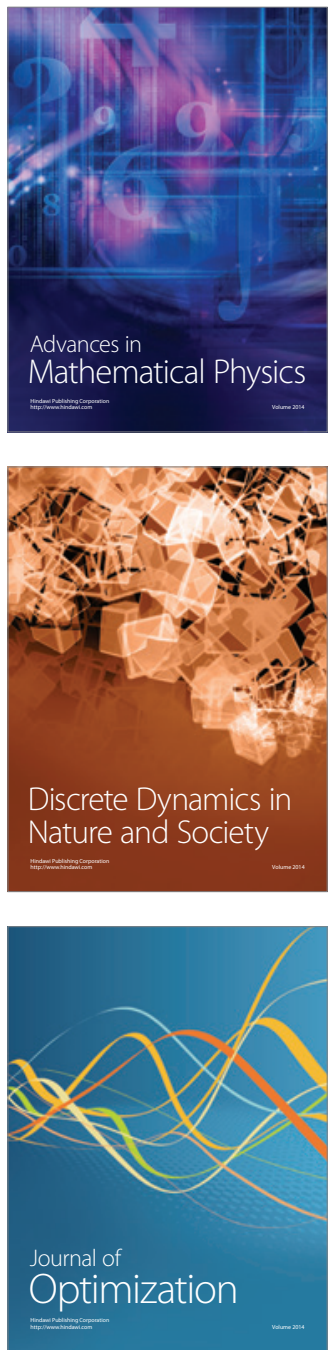